\title{
Broadband Enhancement of Faraday Effect Using Magnetoplasmonic Metasurfaces
}

\author{
Soheila Kharratian, ${ }^{1}$ Hakan Urey, ${ }^{2}$ and Mehmet C. Onbaşl1 1 ,** \\ ${ }^{1}$ Department of Materials Science and Engineering, Koç University, Istanbul, Turkey \\ 2 Department of Electrical and Electronics Engineering, Koç University, Istanbul, Turkey \\ *monbasli@ku.edu.tr
}

\begin{abstract}
Magnetooptical Faraday effect enables ultrafast photonic devices based on nonreciprocal polarization rotation; however, the intrinsic weakness of Faraday effect prevents miniaturization and practical applications of nonreciprocal photonic devices. Magnetoplasmonics offers new mechanisms for enhancing magnetooptical effects using surface plasmon resonances, which generally have narrow bandwidths. Using finite-difference time-domain modelling, we demonstrate a magnetoplasmonic metasurface, which remarkably enhances the Faraday effect in a wide spectral range. While Faraday rotation in a bare bismuth-substituted yttrium iron garnet film is below $0.02^{\circ}$ in the studied range of 600-1600 $\mathrm{nm}$, the proposed metasurface yields few degrees of rotation in a broad band with a maximum exceeding $6.5^{\circ}$, which indicates about three orders of magnitude enhancement. We also show that by optimizing the configuration of the system including the geometry and excitation parameters, the metasurface response and operation band can be tuned further, and rotation values higher than $20^{\circ}$ can be achieved. Finally, we present guidelines for designing magnetoplasmonic metasurfaces.
\end{abstract}

Keywords: Magnetooptics, Plasmonics, Faraday effect, Metasurfaces, Faraday effect

\section{Introduction}

Magnetooptical (MO) Faraday effect is the rotation of the polarization plane of light when it passes through a magnetized matter. The angle of rotation is directly proportional to the path length of the light in the magnetized medium, with a coefficient of proportionality named specific Faraday rotation:

$\alpha_{F}=\frac{\chi}{\mathrm{n}} \overrightarrow{\mathrm{M}} \cdot \overrightarrow{\mathrm{k}}$

where $\alpha_{F}$ is the Faraday rotation per unit path length, $\chi$ is the magnetic susceptibility, $\mathrm{n}$ is the refractive index, $\overrightarrow{\mathrm{M}}$ is the magnetization vector, and $\overrightarrow{\mathrm{k}}$ is the wavevector $[1,2]$. Faraday effect finds applications in various optical devices including isolators [3-5], circulators [6-8], and spatial light modulators [9-11], as well as data storage [12-14], sensing/imaging systems [15-17], and in the growing field of spintronics [18, 19]. Despite these applications, the small magnitude of the specific Faraday rotation in the majority of MO materials limits its applicability and prevents miniaturization of the MO devices [20]. Hence, enhancement of the Faraday effect has become an attractive research topic and different approaches such as using suspended plasmonic particles [21, 22], core-shell magnetoplasmonic nanoparticles [23, 24], magnetoplasmonic crystals [25-27], magnetophotonic crystals [28-30], and graphene infiltration [31] have been reported in this regard.

Plasmonic metasurfaces (PMs) present another method for enhancing MO effects, particularly in the visible and nearinfrared range. PMs are two-dimensional arrays of metallic nanoantennas with subwavelength spacings that induce oscillations of the conduction electron density, called surface plasmons (SPs), during interaction with an electromagnetic (EM) wave [32-36]. When the EM wave is coupled to the SPs, coherent fields with mixed nature of EM waves and surface charges, named surface plasmons polaritons (SPPs), are formed in the metal-dielectric interface. SPPs propagate along the interface and decay evanescently in the perpendicular direction due to the damping by the Joule heating and radiative emission. Thus, SPPs confine the EM waves along the metal-dielectric interface, in length scales on the order of diffraction limit $\left(\sim \lambda_{0} / 2 n\right)$. The integration of PMs into MO materials allows for localization of EM wave in the MO material, which increases light-matter interactions and leads to enhanced MO 
effects. Despite the increased interaction, the resonant characteristic of this phenomenon causes the MO enhancement to be generally narrowband and limits the applications [37-42]. Recently, broadband optical modulation has been studied using different approaches such as metamaterials [43-45] or photonic crystals [46]. In the field of magnetooptics, Kalish et al. [47] have demonstrated multiband enhancement of transverse MO Kerr effect (TMOKE) using quasiperiodic plasmonic structures. Pappas et al. [48] have reported a relatively broadband enhancement of longitudinal MO Kerr effect (LMOKE) in the spectral range of 600-750 nm, by embedding gold nanoparticles into bismuth-substituted yttrium iron garnet (Bi:YIG) films. Nevertheless, an effective broadband enhancement of the Faraday effect has not previously been reported, whereas Faraday rotation is potentially the largest and most practical MO effect.

In this study, using finite-difference time-domain (FDTD) simulations, we demonstrate a magnetoplasmonic metasurface comprised of a 2D array of gold nanoantennas on a MO Bi:YIG film, which remarkably enhances the Faraday effect in a wide spectral range over the near-infrared band. The proposed structure entails different periodic distances, which lead to multiple resonances, and the overlap of these resonances enables a broadband response. First, we compare the transmission (T) and Faraday rotation (FR) spectra of the proposed metasurface with those of the bare Bi:YIG film and the Bi:YIG film covered by a uniform gold layer. Next, we study the mode profiles in the peaks and dips of the spectra and discuss the origin of the resonances and MO enhancement. We also investigate the effect of geometric and excitation factors, such as width and thickness of the plasmonic nanoantennas, periodic lattice parameters, and polarization of the incident light on the T and FR spectra. Finally, we discuss the feasibility of active tunability and present guidelines for designing magnetoplasmonic metasurfaces.

\section{Structure, materials, and methods}

The proposed magnetoplasmonic metasurface consists of a 2D array of ultrathin gold (Au) nanoantennas on a $10 \mathrm{~nm}$ thick Bi:YIG film deposited on a glass substrate, as shown in Fig. 1. Au is a noble metal with small Ohmic losses and low absorption, which offers highly functional plasmonic properties [49], and Bi:YIG is an MO material with large Verdet constant that yields large MO effects in relatively low magnetic fields [50]. The geometric parameters $\mathbf{l}, \mathbf{s}$, and $\mathbf{d}$ in Fig. 1 have fixed values of 250, 80, and $50 \mathrm{~nm}$, respectively. Width (w), thickness (t), the period of structure in $\mathrm{x}$ and y directions ( $\mathbf{p}_{\mathbf{x}}$ and $\mathbf{p}_{\mathbf{y}}$ ) were initially considered to be 50,5,250, and $400 \mathrm{~nm}$, respectively, however, the effects of changing these structural parameters on the optical and magnetooptical response of the metasurface are studied in Section 4. The incident light was first assumed to be polarized in the $\mathrm{x}$ direction $\left(\boldsymbol{\theta}=0^{\circ}\right)$, but the effect of the exciting polarization is also investigated as a tuning mechanism for the metasurface response.

Metasurfaces with extremely small thicknesses are crucial for the miniaturization of optoelectronic devices for compact applications, e.g. certain sensing, robotic, and space systems, or studying light-matter interactions at the nanoscale [51, 52]. Reduction of thickness in thin films leads to new dimensionality-related effects originating from strong confinement of electrons, and ultrathin films exhibit peculiar properties due to the quantum size effect. While plasma frequency $\left(\omega_{\mathrm{p}}\right)$ in bulk materials and rather thicker films is only defined by the electronic band structure of the material, $\omega_{\mathrm{p}}$ in ultrathin films shows a redshift with decreasing thickness. Such a tunability introduces a unique platform to design advanced multifunctional devices in a wide operational range. Furthermore, smaller thicknesses cause lower dissipative losses and retain low optical damping, which makes them favorable in terms of energy considerations and support of strong plasmons for a device design [51, 53]. In gold clusters with sizes $>3 \mathrm{~nm}$, the optical properties can be described by a bulk dielectric function with relevant modifications corresponding to the electron scattering at the surface. Below this size, a gradual discretization occurs in the plasmonic resonances [54]. Although controlling the thickness in the nanometric scale is a challenging measure, ultrathin films with thicknesses smaller than $10 \mathrm{~nm}$ down to atomic layers have been studied for their strong and tunable plasmonic effects [55-59].

We use the finite-difference time-domain (FDTD) method and Lumerical software to calculate the field components and investigate the optical and $\mathrm{MO}$ response. The optical data for gold and glass $\left(\mathrm{SiO}_{2}\right)$ are taken from Johnson and Christy [60] and Palik [61], respectively. Considering the magnetization (M) direction perpendicular to the film plane (xy), i.e. M parallel to $\mathrm{z}$ direction, the MO film is defined by a permittivity tensor in the form of: 
$\varepsilon=\left[\begin{array}{ccc}\varepsilon_{1} & +i \varepsilon_{2} & 0 \\ -i \varepsilon_{2} & \varepsilon_{1} & 0 \\ 0 & 0 & \varepsilon_{1}\end{array}\right]$

where the diagonal element $\varepsilon_{1}$ is related to the complex refractive index and the off-diagonal element $\varepsilon_{2}$ is responsible for the MO activity such as Faraday/Kerr rotation and ellipticity. Generally, $\varepsilon_{1}$ and $\varepsilon_{2}$ have complex values, which are dispersive with respect to wavelength. Here, we use the average values of $\varepsilon_{1}=5.5+0.0025 \mathrm{i}$ and $\varepsilon_{2}=(1-0.15 \mathrm{i}) \times 10^{-2}$ for Bi:YIG [40, 41, 62-64].

(a)

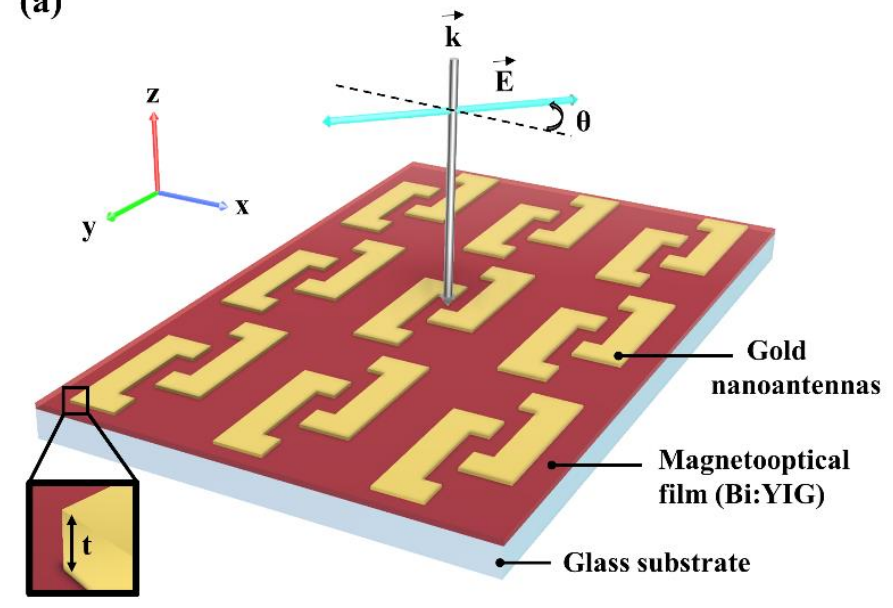

(b)

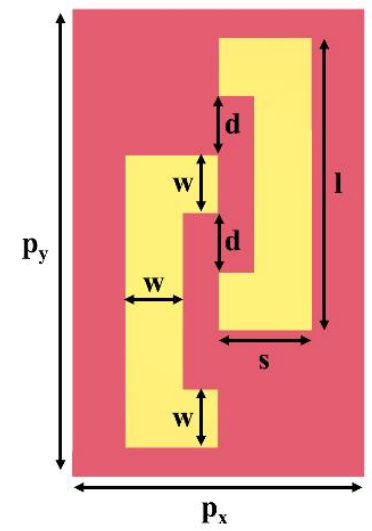

Fig. 1. (a) Schematic layout of the proposed magnetoplasmonic metasurface. The thickness of the magnetooptical Bi:YIG film is $10 \mathrm{~nm}$. (b) Top view of a unit cell, where the geometric parameters $\mathbf{I}=250, \mathbf{s}=80$, and $\mathbf{d}=50 \mathrm{~nm}$ have fixed values throughout the study. The width $(\mathbf{w})$, thickness $(\mathbf{t})$, the period of the structure in $\mathrm{x}$ and $\mathrm{y}$ directions $\left(\mathbf{p}_{\mathbf{x}}\right.$ and $\mathbf{p}_{\mathbf{y}}$ ) were initially considered to be $50,5,250$, and $400 \mathrm{~nm}$, respectively, however, the effects of changing these variables on the response of the magnetoplasmonic metasurface are studied. The incident light was first assumed to be $\mathrm{x}$-polarized $\left(\boldsymbol{\theta}=0^{\circ}\right)$, but polarization variation is investigated as a mechanism for tuning the response of the metasurface.

FDTD simulations are performed in a unit cell with periodic boundary conditions in $\mathrm{x}$ and $\mathrm{y}$ directions and perfectly matched layer (PML) boundary conditions in the $\mathrm{z}$ direction. A planar light source with linear polarization and propagating perpendicular to the film plane, along $-\mathrm{z}$ axis, is used.

\section{Theory}

The photon momentum of a plasmon on a thin metal film is generally several hundred times larger than the momentum of a free space photon with the same energy [65]. Thus, for efficient coupling of light to SPs and exciting SPPs, a momentum matching technique such as using a prism, isolated holes, or gratings is required [42, 66]. When the plasmonic structure has a periodic configuration, based on the unit cell boundary conditions, different complicated phenomena can occur along with the excitation of SPPs. In structures that have continuous metal-dielectric interfaces in the unit cell boundaries, such as 1D and 2D plasmonic gratings, a collective radiative effect named SPP-Bloch wave (SPP-Bw) mode can be observed. According to the Bragg coupling condition, SPP-Bw mode is excited when:

$\overrightarrow{\mathrm{k}}=\overrightarrow{\mathrm{k}} \|+\overrightarrow{\mathrm{G}}$

where $\overrightarrow{\mathrm{k}} \|$ is the in-plane wavevector of the incident light, and $\overrightarrow{\mathrm{G}}$ is the reciprocal lattice vector. 
For a plasmonic metasurface consisted of a periodic array of discrete nanoantennas with large dielectric spacings, a collective lattice plasmon mode caused by scattering from the array of nanoantennas and mediated by the diffractive coupling between SPPs can be generated. In this case, the optical energy scattered by one antenna is more likely to be collected by the neighboring antennas, resulting in a collective lattice plasmon instead of decaying as free-space light. The lattice plasmon modes can be considered as a type of surface Bloch mode composed of many Bloch harmonics from the array. According to the Bloch's theorem, for a square 2D array, the in-plane wavevector for the diffraction order of $\{m, n\}$ is defined as:

$\overrightarrow{\mathrm{k}}_{\mathrm{m}, \mathrm{n}}=\overrightarrow{\mathrm{k}}_{0} \sin \varphi+\mathrm{m} \frac{2 \pi}{\mathrm{p}_{1}} \overrightarrow{\mathrm{u}}_{1}+\mathrm{n} \frac{2 \pi}{\mathrm{p}_{2}} \overrightarrow{\mathrm{u}}_{2}$

where $\overrightarrow{\mathrm{k}}_{0}$ is the free space wavevector, $\varphi$ is the incidence angle, $\mathrm{p}_{1}$ and $\mathrm{p}_{2}$ are the array pitches in the directions of unit vectors $\overrightarrow{\mathrm{u}}_{1}$ and $\overrightarrow{\mathrm{u}}_{2}$, respectively. When all the momentum of the scattered radiation is parallel to the plane of the array,

i.e. $\left|\vec{k}_{m, n}\right|=\vec{k}_{0}$, and the normal wavevector vanishes, the effect is called Rayleigh's anomaly (RA). This phenomenon converts the scattered fields into grazing waves that can be hybridized with the SPPs and result in SPP-RA modes. RA is not a resonant effect, however, the SPP-RA coupling can lead to sharp resonant features in the spectra and offers a mechanism for enhancing SPP modes. These hybridized resonances are called surface lattice resonances (SLRs).

Another mechanism for enhancing SPP modes is the hybridization of plasmonic modes with guided modes when the plasmonic nanoantennas are in proximity to waveguiding structures. In such a configuration, the antenna array couple light from free space into the waveguide modes. Since such couplers can also decouple light from a waveguide mode into free space, these modes are leaky and known as quasi-guided modes. SLRs are favored for large plasmonic antennas in a surrounding medium with homogeneous dielectric properties, while the quasi-guided modes require a waveguiding component with a higher refractive index compared to the surrounding environment $[37,49,67,68]$.

In summary, the spectral and spatial overlap of plasmonic and photonic modes lead to superior hybridized modes combining deep subwavelength confinement and strong light-matter interaction characteristic with long-range guiding and modal volume extended over film plane. These plasmonic-photonic modes enable sharp optical resonances that can be tuned by geometric, lattice, and excitation parameters.

\section{Results and discussion}

In this section, the results on the broadband enhancement of Faraday rotation using the magnetoplasmonic metasurfaces are presented and the origin of the enhancement is systematically investigated. Fig. 2 compares the T and FR spectra of the proposed magnetoplasmonic metasurface (red lines) with those of a bare Bi:YIG film (black lines) and the Bi:YIG film covered by a plain gold layer with the same thickness as the nanoantennas' thickness (blue lines), all on glass substrates. The geometric variables are $\mathbf{w}=50 \mathrm{~nm}, \mathbf{t}=5 \mathrm{~nm}, \mathbf{p}_{\mathbf{x}}=250 \mathrm{~nm}$, and $\mathbf{p}_{\mathbf{y}}=400 \mathrm{~nm}$, and an $\mathrm{x}$-polarized light source $\left(\boldsymbol{\theta}=0^{\circ}\right)$ is used for the metasurface investigated in this figure. The T spectrum of Bi:YIG film does not change significantly within the studied range of 600-1600 nm. The plasmonic metasurface, however, shows resonant features, and the transmission of the Bi:YIG film with a plain gold layer diminishes almost linearly with increasing wavelength (Fig. 2a). Comparison of the FR spectra (Fig. 2b) shows negligible FR values for the bare Bi:YIG film in comparison to the proposed metasurface, and adding a plain gold layer on top of the Bi:YIG film improves the Faraday effect only slightly in the wavelengths shorter than $1400 \mathrm{~nm}$, as magnified in Fig. 2c. While the FR values for the Bi:YIG film are below $0.02^{\circ}$ in the studied spectral range, rotation values up to $6.5^{\circ}$ are achievable using the proposed metasurface as shown in Fig. $2 b$. 

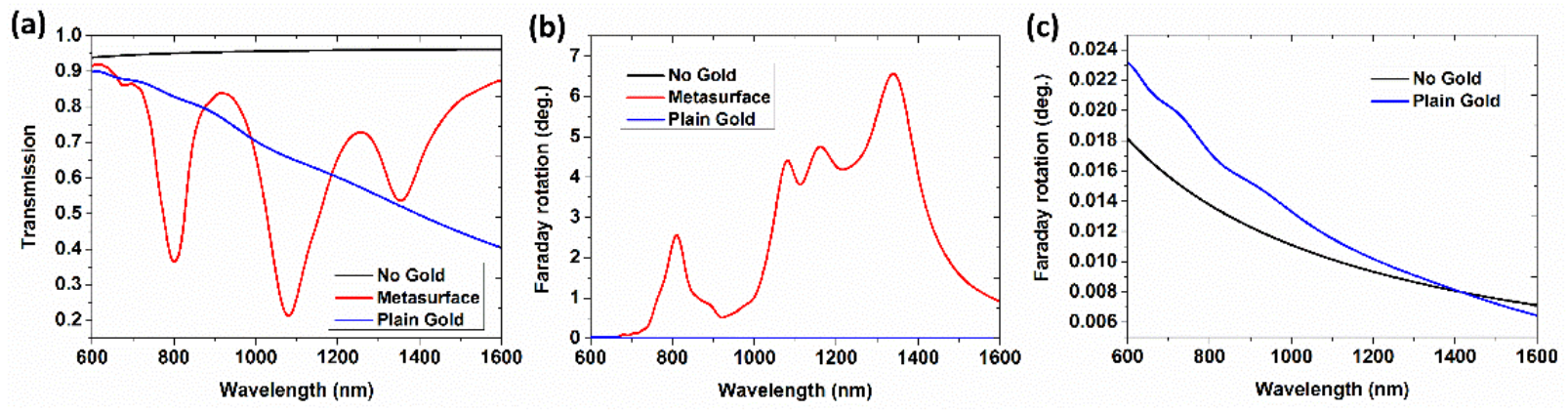

Fig. 2. (a) Transmission and (b) Faraday rotation spectra for a $10 \mathrm{~nm}$ thick Bi:YIG film (black lines), the plasmonic metasurface comprised of $10 \mathrm{~nm}$ thick Bi:YIG film and $5 \mathrm{~nm}$ thick plasmonic structures with geometric parameters of $\mathbf{w}=50, \mathbf{p}_{\mathbf{x}}=250$, and $\mathbf{p}_{\mathbf{y}}=400$ (red lines), and a $10 \mathrm{~nm}$ thick Bi:YIG film covered by a $5 \mathrm{~nm}$ thick gold film on top (blue lines). (c) Magnification of part (b) for small rotation angles. The polarization of the light source is considered to be in the $\mathrm{x}$ direction $\left(\boldsymbol{\theta}=0^{\circ}\right)$ for these calculations.

The structure of the proposed metasurface comprises different periodic distances as shown in Fig. S1 (Online Resource 1), which leads to multiple resonant peaks in the T and FR spectra. The X-polarized incident light experiences four different periodic configurations on the metasurface, each inducing a resonance in the metasurface response [69]. As a result, four major peaks are observed in the FR spectrum of the magnetoplasmonic metasurface (Fig. 2b). If one looks at the structure as a simplified 1D grating, one can see four different square wave patterns (1-4). This results in four different energy distributions among harmonics in k-space. As a result, one can expect four different resonant peaks. The overlap of these resonances leads to enhanced FR in a broad spectral range. The FR peaks around $\lambda=800$, 1080, and $1355 \mathrm{~nm}$ are correlated with the respective dips in the T spectrum. For the FR peak around $1160 \mathrm{~nm}$, the related transmission dip is indeed covered by the adjacent stronger dip at $1080 \mathrm{~nm}$, as can be understood from the asymmetric shape of this resonant dip in the $\mathrm{T}$ spectrum (Fig. 2a). The proposed plasmonic metasurface makes it possible to achieve few degrees of rotation in a broad wavelength range and a maximum FR exceeding $6.5^{\circ}$, in a total thickness of $15 \mathrm{~nm}$, which indicates about three orders of magnitude enhancement in Faraday effect at the respective wavelength.

To explain the resonant features of the magnetoplasmonic metasurface observed in Fig. 2, we investigate the mode profiles at the main peaks and dips of its spectra. Fig. 3 shows the distribution of the electric field normalized to the incident field, $\left|\mathrm{E} / \mathrm{E}_{0}\right|$, at the interface between the plasmonic structures and the MO film, for different wavelengths. At $\lambda=680 \mathrm{~nm}$, a minor dip in the T spectrum is observed, accompanied by a minute peak in the FR spectrum. The mode profile of a unit cell at this wavelength shows modes propagating along the gold antennas, most pronouncedly in the shorter edges, and the enhancement factor $\left(\left|\mathrm{E}_{\mathrm{E}} \mathrm{E}_{0}\right|\right)$ is mainly below 10 (Fig. 3a). At $\lambda=800 \mathrm{~nm}$, SPPs are mostly propagating along the longer edges of the nanoantennas (Fig. 3b) with some localization under the gold structures. At $\lambda=915 \mathrm{~nm}$, the propagating surface plasmon resonance (PSPR) mode intensity diminishes (Fig. 3c). Comparison of parts $b$ and $c$ of Fig. 3 shows similar values for the maximum field enhancement (about 28) at both wavelengths, however, distribution of the high field in a larger area at $\lambda=800 \mathrm{~nm}$ leads to a greater net enhancement at this wavelength and places it in a peak position in FR spectrum, while $\lambda=915$ represents a dip. At $\lambda=1080$ and $1255 \mathrm{~nm}$, the surface plasmons are mostly localized in the corners of the nanoantennas (Fig. 3d,e). These strongly localized surface plasmon resonances (LSPRs) increase the light-matter interactions and result in remarkable enhancement of FR. Higher enhancement factors in Fig. 3d compared to Fig. 3e leads to an FR peak at $1080 \mathrm{~nm}$ and a relative decrease at $1255 \mathrm{~nm}$, yet showing a significant rotation value. At $\lambda=1355 \mathrm{~nm}$, both PSPR and LSPR modes coexist (Fig. 3f), leading to a maximum FR, although the maximum E-field enhancement is not observed at this wavelength. 

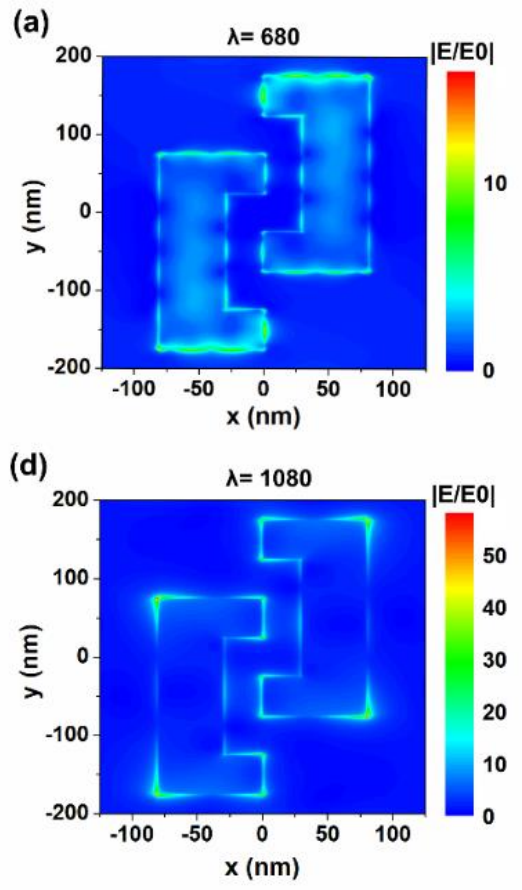
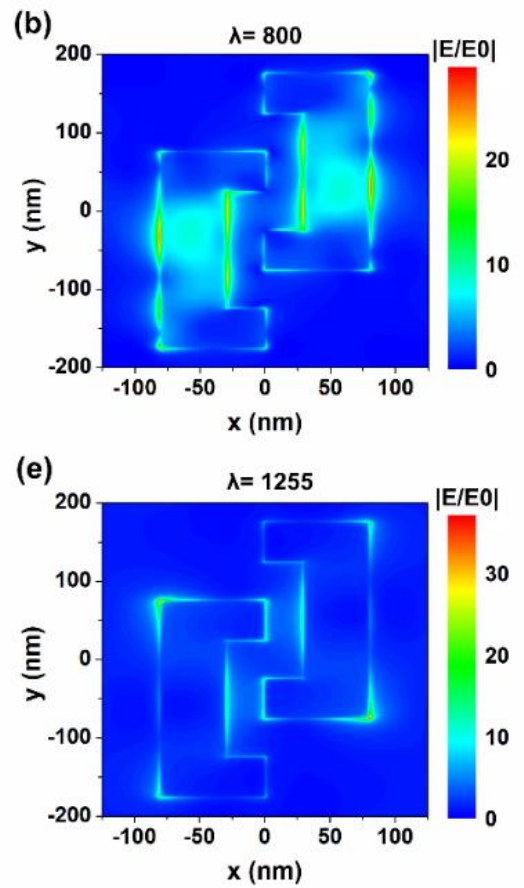
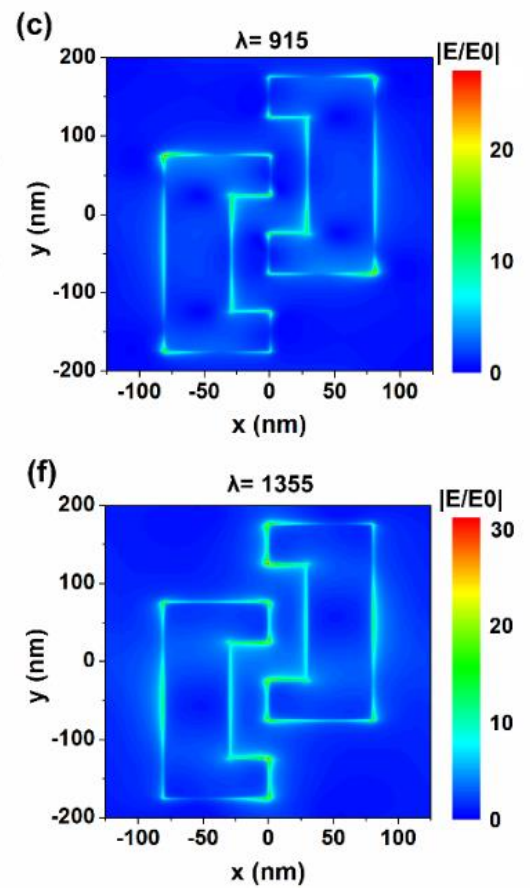

Fig. 3. Distribution of the electric field normalized to the incident field, $\left|\mathrm{E} / \mathrm{E}_{0}\right|$ in the $x y$ plane at the interface between the gold nanoantennas and the Bi:YIG film, for different wavelengths of (a) $\lambda=680 \mathrm{~nm}$, (b) $\lambda=800 \mathrm{~nm}$, (c) $\lambda=915$ $\mathrm{nm}$, (d) $\lambda=1080 \mathrm{~nm}$, (e) $\lambda=1255 \mathrm{~nm}$, and (f) $\lambda=1355 \mathrm{~nm}$. Note the different maxima among the color bars.

The effect of geometric parameters on the spectral $\mathrm{T}$ and $\mathrm{FR}$ response of the plasmonic metasurface has been studied in Fig. 4. Increasing the width of gold nanostructures ( $\mathbf{w})$ reduces the transmission, improves the FR, and causes a redshift in the spectra, as shown in Fig. 4a,b. Giant rotation angles, higher than $20^{\circ}$, are achieved for $\mathbf{w}=70 \mathrm{~nm}$. As indicated in Fig. 4c,d, increasing the thickness of gold nanostructures (t), not only decreases the transmission and enhances the FR but also considerably blueshifts the spectra. For thicknesses smaller than $5 \mathrm{~nm}$, no significant FR peaks are observed in the studied band. Increasing $\mathbf{t}$ sharpens the peaks and narrows down the spectral range of enhancement. While both $\mathbf{w}$ and $\mathbf{t}$ can be used as tools of tuning the operation wavelength, peak positions are more sensitive to the change of $\mathbf{t}$. Knowing the effects of these geometric parameters, one can optimize the proposed magnetoplasmonic metasurface based on the application requirements and the limits for $\mathrm{T}$ and FR. According to the results depicted in Fig. 4, for the broadband applications, plasmonic structures with large widths and small thicknesses are recommended. 
(a)

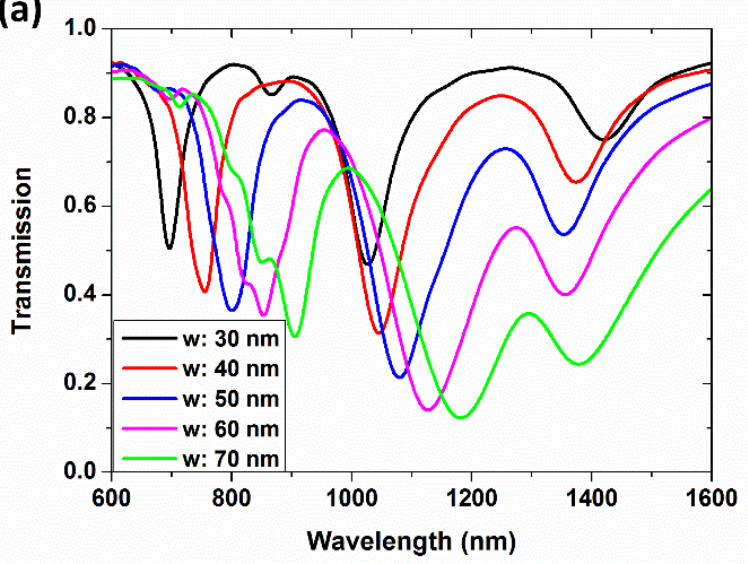

(c)

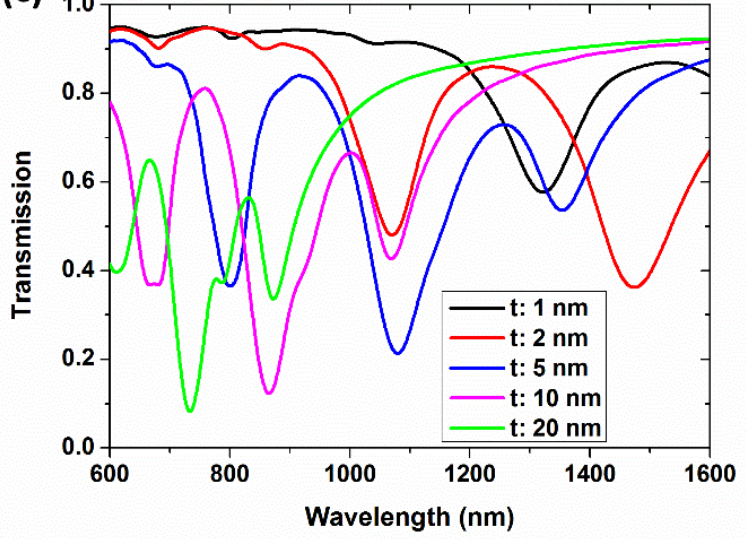

(b)

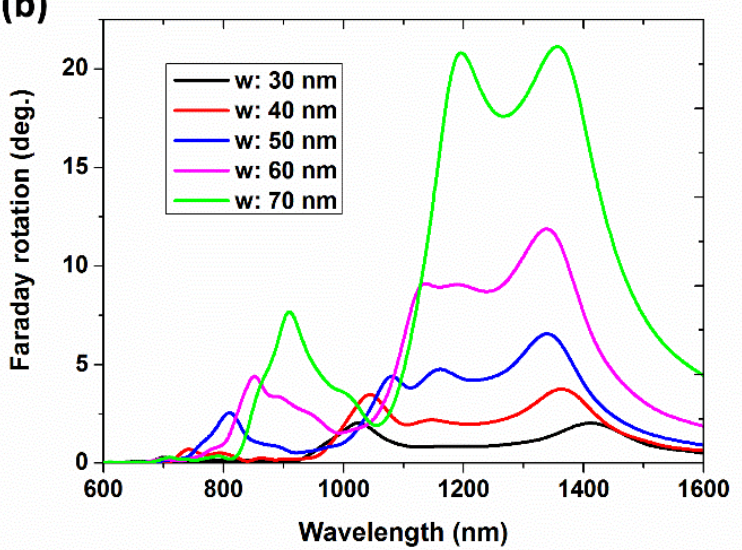

(d)

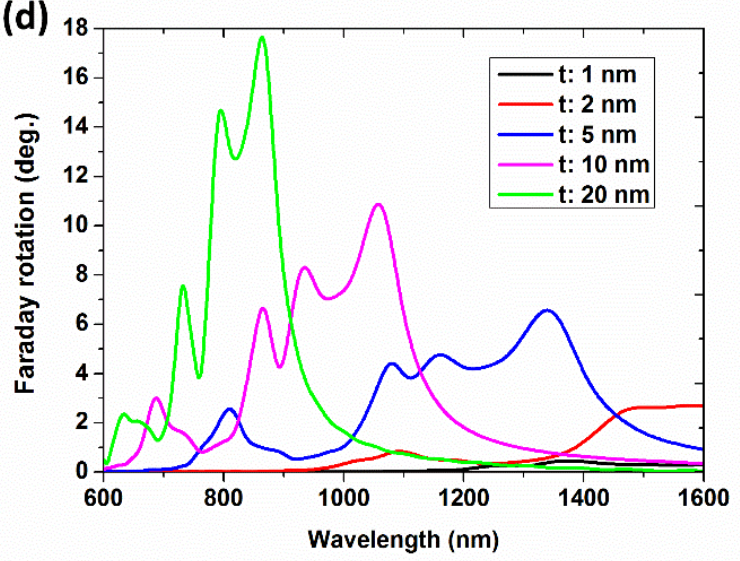

Fig. 4. Effect of $(a, b)$ width and $(c, d)$ thickness of the gold nanoantennas on the optical and magnetooptical responses of the magnetoplasmonic metasurface.

Considering the metasurface with $\mathrm{w}=70 \mathrm{~nm}$ in Fig. 4a,b, three distinctive resonances are recognized. In contrast to the resonances around $\lambda=900$ and $1200 \mathrm{~nm}$ that blueshift with decreasing the antenna sizes, the resonance around $\lambda$ $=1350 \mathrm{~nm}$ shows no significant spectral shift with changes in the size of the plasmonic structures. Hence, the resonances around 900 and $1200 \mathrm{~nm}$ can be assigned to plasmonic modes and the resonance at $1350 \mathrm{~nm}$ can be related to photonic modes of the metasurface. In this case, where the thickness of the nanoantenna is considered to be $5 \mathrm{~nm}$, a pronounced coupling is not observed between the modes. However, for higher thicknesses (purple and green lines in Fig. 4c,d), a strong coupling exists between the plasmonic and photonic modes, and in accordance with the previous reports [58], all the resonances considerably shift towards shorter wavelengths with increasing $\mathbf{t}$. Such a strong coupling results in sharp resonances, enabling up to $18^{\circ} \mathrm{FR}$ for nanoantennas with $20 \mathrm{~nm}$ thickness.

In Fig. 5, we investigate the impact of periodicity on the performance of the proposed metasurface. Increasing the period of the plasmonic structures in the $\mathrm{x}$ direction $\left(\mathbf{p}_{\mathbf{x}}\right)$, leads to higher transmission but lower FR (Fig. 5a,b). Increasing the period in the y direction $\left(\mathbf{p}_{\mathbf{y}}\right)$, has a similar effect, however, in a less pronounced manner (Fig. $\left.5 \mathrm{c}, \mathrm{d}\right)$. In contrast to the geometric parameters that provide a means to control the spectral position of the resonant peaks in addition to the peak intensity, periodicity does not noticeably change the shape of spectra or position of the peaks. Thus, for enhancing the FR without changing the working wavelengths, reducing the period of the plasmonic array would be the best reconfiguration option. 
(a)

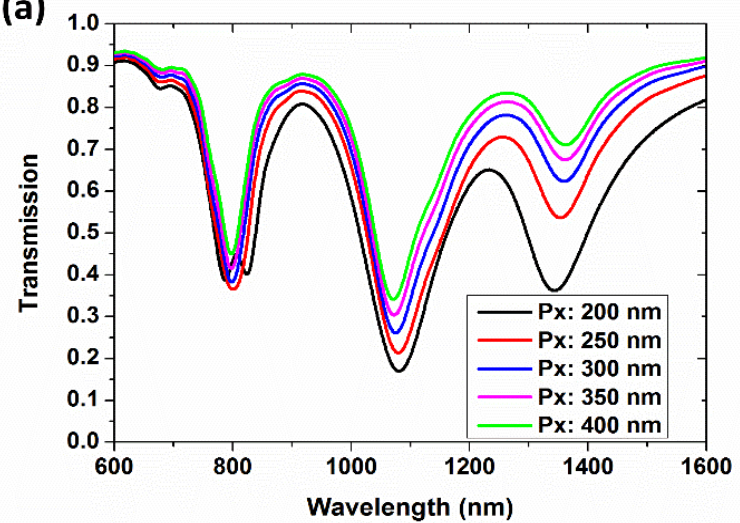

(c)

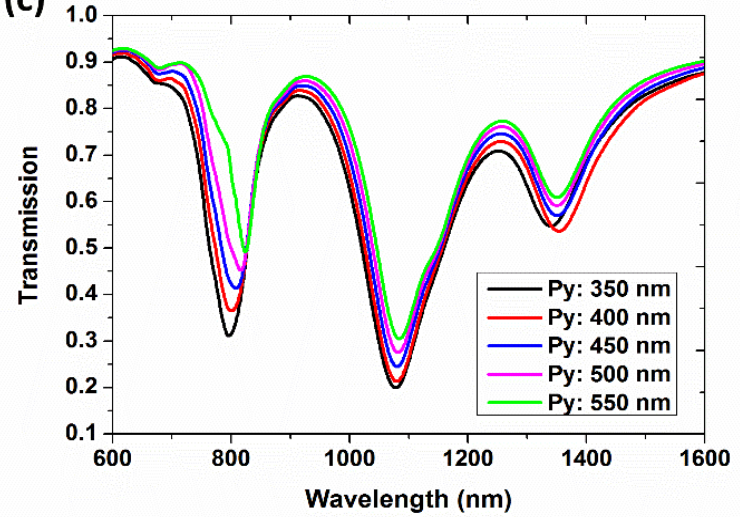

(b)

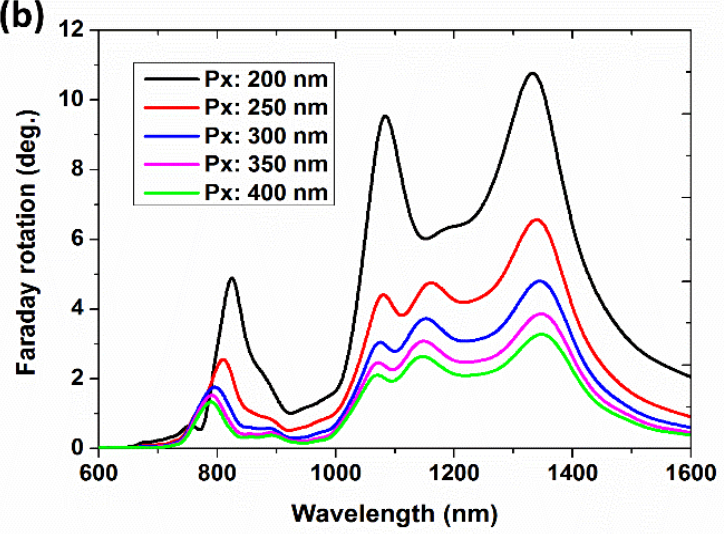

(d)

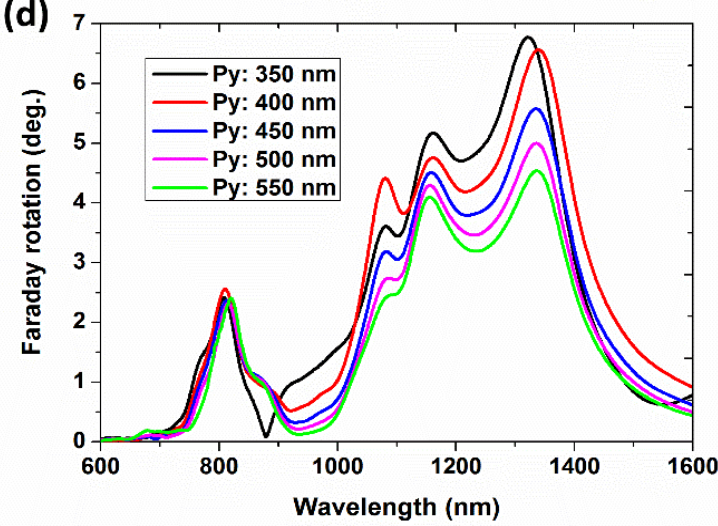

Fig. 5. Effect of periodicity in $(\mathrm{a}, \mathrm{b}) \mathrm{x}$ direction and $(\mathrm{c}, \mathrm{d}) \mathrm{y}$ direction on the optical and magnetooptical responses of the magnetoplasmonic metasurface.

In addition to the structural parameters that need to be considered before design and fabrication, there are other parameters, which can dynamically tune the performance of a metasurface. A means for such a control is the polarization of the incident light. Fig. 6 indicates the effect of the polarization direction of light on the optical and magnetooptical responses of the magnetoplasmonic metasurface. According to Fig. 6a, by changing the polarization of the incident light from $\mathrm{x}$ towards y direction (increasing $\boldsymbol{\theta}$ from $0^{\circ}$ to $90^{\circ}$ ), a remarkable increase in transmission accompanied by changes in the shape of $\mathrm{T}$ spectra is observed. Fig. $6 \mathrm{~b}$ indicates that the metasurface has a broadband enhancement effect only for right-angle polarizations $\left(\boldsymbol{\theta}=0^{\circ}\right.$ and $\left.90^{\circ}\right)$ and oblique polarization angles exhibit strong and narrow resonances. It is notable that for oblique polarization, the sign of FR changes with asymmetric Fano-type line shapes. In such a configuration, the structural asymmetries allow for the coupling of discrete non-radiative (dark) modes that result from SLRs and have finite spectral linewidths, with the normal transmittance through the film (bright mode) that can be considered as a continuum. The interference between dark and bright modes results in Fano resonances that have narrow spectral width and enable more efficient confinement of light, leading to a large enhancement of optical field and FR [37, 41, 67, 68]. Fig. 6 shows that for incident light with $\boldsymbol{\theta}=30^{\circ}$, FR angles up to about $20^{\circ}$ are achieved with reasonably high transmissions (over $40 \%$ ). Comparing the spectra for $\boldsymbol{\theta}=0^{\circ}$ and $90^{\circ}$, one can observe that y-polarized light results in slightly smaller FR while its transmission is significantly higher than the light with x polarization. Therefore, for the applications that are more sensitive to transmission, using a y-polarized light source with the proposed metasurface is preferable. 
(a)

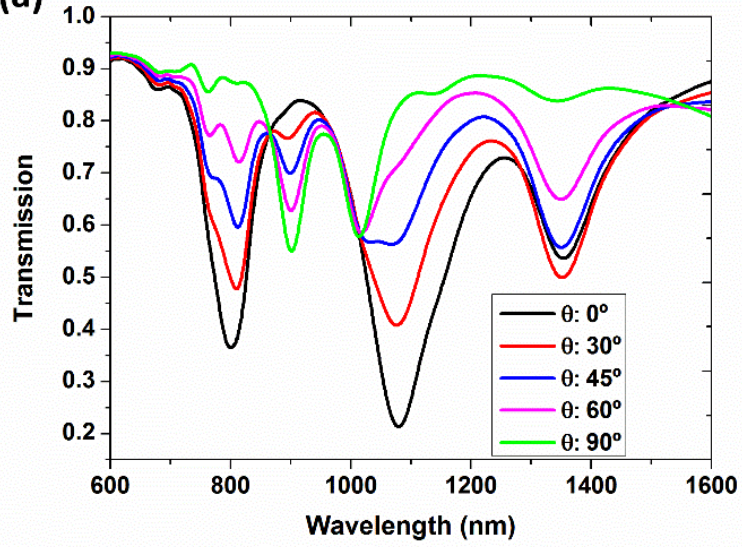

(b)

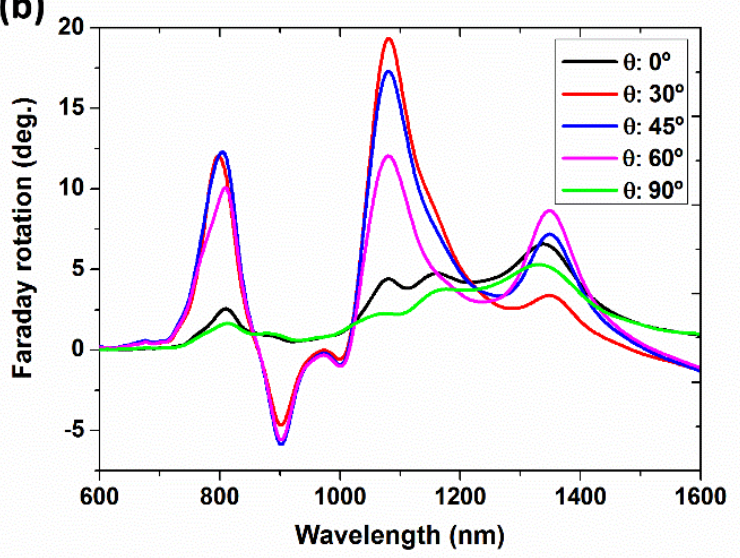

Fig. 6. Effect of polarization of light on (a) transmission and (b) Faraday rotation of the magnetoplasmonic surface. $\boldsymbol{\theta}$ is the angle between the polarization plane of the incident light and the $\mathrm{x}$ axis.

Another mechanism for tuning the performance of magnetoplasmonic metasurface is the use of a magnetic field as an external signal for dynamic control of the magnetooptical response. At a given configuration, altering the magnetization of the MO material would change the off-diagonal element of the permittivity tensor, $\varepsilon_{2}$, which determines the magnitude of achieved FR [70]. Switching the magnetization direction would change the sign of FR, leading to the MO rotation in opposite angular orientation [71].

\section{Magnetoplasmonic metasurface design guidelines}

In this section, based on the results shown in Fig. 2-6, we present guidelines for designing magnetoplasmonic metasurfaces with any desired properties:

\subsection{Operation wavelengths}

The application requirements determine the operation wavelengths. The MO material must have nonzero and sufficiently large off-diagonal elements in the permittivity tensor, at the application wavelengths. The operation band of plasmons determines the plasma frequency and the corresponding plasmonic material, e.g. gold and doped graphene for $\mathrm{THz}$ regime [72, 73]. The size of plasmonic antennas has a prominent role in determining the resonance wavelengths. Increasing the width of antennas shifts the peaks towards higher wavelengths, while changing the thickness has an opposite effect. For obtaining multi- or broadband performance, the geometry of the plasmonic nanoantennas must include different periodic distances. The geometrical configuration, especially the thickness of the nanoantennas, determines the span of the operational band for the plasmonic metasurface.

\subsection{Simultaneous maximization of the magnetooptical effect and transmission}

An efficient plasmonic metasurface for nonreciprocal devices requires materials with large MO properties and low optical losses. Simultaneous enhancement of transmission and Faraday rotation is necessary for achieving an acceptable signal-to-noise ratio in applications such as telecommunications, sensing, and signal processing. Both localized and propagating surface plasmon modes, and also coupling between plasmonic and photonic modes could be exploited for maximizing the FR enhancement while controlling the losses. Multiple resonances in the transmission spectra lead to strong multi- or broadband enhancement of MO effects. The configurational parameters provide tuning means for the optimization of optical and MO responses of the magnetoplasmonic metasurface. The results presented in this study show that the thickness of nanoantennas $(\mathbf{t})$ and the periodicity of structure in the direction of the incident light polarization (e.g. $\mathbf{p}_{\mathbf{x}}$ in the case of $\mathrm{x}$-polarized incident light) allow for significant enhancement of FR without a 
remarkable drop in T. Moreover, for the proposed metasurface, y-polarized incident light yields transmission values considerably higher than the x-polarized light, while the resultant FR is only slightly lower.

\subsection{Polarization dependence/independence}

For polarization or angular orientation selectivity/insensitivity of the magnetoplasmonic metasurface, the geometry of nanoantennas must be designed without/with $\mathrm{n}$-fold rotational and mirror symmetries. In other words, mirror plane and circular symmetries in the nanoantenna geometry could help achieve polarization independence, whereas the lack of symmetry in the antennas structure leads to different responses based on the polarization of the incident light. The latter enables a new class of optical devices that can be switched and controlled by the polarization of light [74].

\section{Conclusion}

A magnetoplasmonic metasurface was demonstrated using a two-dimensional array of gold nanoantennas on a magnetooptical Bi:YIG film, which remarkably enhances the Faraday effect in a wide near-infrared band. While Faraday rotation in the bare Bi:YIG film is below $0.02^{\circ}$ within the studied wavelength range of $600-1600 \mathrm{~nm}$, the proposed metasurface significantly improves the performance. The metasurface enables few degrees of rotation in the broad band of 1000-1400 $\mathrm{nm}$ with a maximum exceeding $6.5^{\circ}$. This indicates about three orders of magnitude enhancement for the Faraday effect, in a total thickness of $15 \mathrm{~nm}$ (comprised of 10-nm thick Bi:YIG film and 5-nm thick gold nanoantennas). The results were further improved by tailoring the geometric parameters and periodicity of the plasmonic structure, leading to rotation angles higher than $20^{\circ}$. Besides the structural parameters that determine the operation wavelengths, the polarization of the incident light and magnetization state of the magnetooptical material were suggested as tools for active tuning of the metasurface response. These tunable magnetoplasmonic metasurfaces could be used for wavelength-selective sensing surfaces, isolators, filters, and other free-space optical signal processing applications. Finally, guidelines based on the investigated results were presented for designing desired magnetoplasmonic metasurfaces and improving the efficiency.

\section{Funding}

This work was financially supported by the European Research Council Advanced Grant (ERC-AdG) Wear3D Project No: 340200, TUBITAK Grant No. 119S362, and TÜBA-GEBIP Award by Turkish Academy of Sciences.

\section{Disclosures}

The authors declare no conflicts of interest.

\section{References}

1. Zvezdin AK, Kotov VA (1997) Modern Magnetooptics and Magnetooptical Materials. Taylor \& Francis Group, New York

2. Kimel A V., Kirilyuk A, Hansteen F, et al (2007) Nonthermal optical control of magnetism and ultrafast laserinduced spin dynamics in solids. J Phys: Condens Matter 19:043201. https://doi.org/10.1088/0953$8984 / 19 / 4 / 043201$

3. Takeda H, John S (2008) Compact optical one-way waveguide isolators for photonic-band-gap microchips. Phys Rev A 78:023804. https://doi.org/10.1103/PhysRevA.78.023804

4. Bi L, Hu J, Jiang P, et al (2011) On-chip optical isolation in monolithically integrated non-reciprocal optical resonators. Nat Photonics 5:758-762. https://doi.org/10.1038/nphoton.2011.270

5. Zhang Y, Du Q, Wang C, et al (2019) Monolithic integration of broadband optical isolators for polarizationdiverse silicon photonics. Optica 6:473-478 
6. Wang Z, Fan S (2005) Optical circulators in two-dimensional Magneto-Optical Photonic Crystals. Opt Lett 30:1989-1991

7. Śmigaj W, Romero-Vivas J, Gralak B, et al (2010) Magneto-optical circulator designed for operation in a uniform external magnetic field. Opt Lett 35:568. https://doi.org/10.1364/OL.35.000568

8. Dmitriev V, Kawakatsu MN, de Souza FJM (2012) Compact three-port optical two-dimensional photonic crystal-based circulator of W-format. Opt Lett 37:3192-3194

9. Ross WE, Psaltis D, Anderson RH (1983) Two-dimensional magneto-optic spatial light modulator for signal processing. Opt Eng 22:485-490. https://doi.org/10.1016/B978-0-08-100877-5.00017-7

10. Park J, Inoue M, Cho J, et al (2003) An optical micro-magnetic device: magnetic-spatial light modulator. J. Magn. 8:50-59

11. Takahashi K, Takagi H, Shin KH, et al (2007) Figures of merit of magneto-optic spatial light modulators with magnetophotonic crystals. Phys Stat Sol (C) 4:4536-4539. https://doi.org/10.1002/pssc.200777215

12. Hill B, Sander I, Much G (1977) Magneto-optic memories. Opt Acta (Lond) 24:495-504. https://doi.org/10.1080/713819553

13. Shirakashi Z, Goto T, Takagi H, et al (2017) Reconstruction of non-error magnetic hologram data by magnetic assist recording. Sci Rep 7:12835. https://doi.org/10.1038/s41598-017-12442-z

14. Nakamura Y, Lim PB, Goto T, et al (2019) Development of heat dissipation multilayer media for volumetric magnetic hologram memory. Appl Sci 9:1738. https://doi.org/10.3390/app9091738

15. Lenz JE (1990) A review of magnetic sensors. Proc IEEE 78:973-989

16. Arakelyan S, Galstyan O, Lee H, et al (2016) Direct current imaging using a magneto-optical sensor. Sensors Actuators A 238:397-401. https://doi.org/10.1016/j.sna.2016.01.002

17. Zhang G, Zhang Z, Xu Y, Wang J (2018) High speed magneto-optical imaging system to investigate motion characteristics of arc plasma in enclosed chamber. Opt Express 26:23156-23166

18. Choi GM, Schleife A, Cahill DG (2017) Optical-helicity-driven magnetization dynamics in metallic ferromagnets. Nat Commun 8:15085. https://doi.org/10.1038/ncomms 15085

19. Kozhaev MA, Chernov AI, Sylgacheva DA, et al (2018) Giant peak of the Inverse Faraday effect in the band gap of magnetophotonic microcavity. Sci Rep 8:11435. https://doi.org/10.1038/s41598-018-29294-w

20. Kharratian S, Urey H, Onbaşlı MC (2020) Advanced Materials and Device Architectures for Magnetooptical Spatial Light Modulators. Adv Opt Mater 8:1901381. https://doi.org/10.1002/adom.201901381

21. Hui PM, Stroud D (1987) Theory of Faraday Rotation by Dilute Suspensions of Small Particles. Appl Phys Lett 50:950-952

22. Chen Q, Wang H, Wang Q, Pan Y (2018) Plasmon Enhanced Faraday Rotation in Fe3O4/Ag Ferrofluids for Magneto Optical Sensing Applications. Plasmonics 13:353-363. https://doi.org/10.1007/s11468-017-0606-1

23. Jain PK, Xiao Y, Walsworth R, Cohen AE (2009) Surface Plasmon Resonance Enhanced Magneto-Optics (SuPREMO): Faraday Rotation Enhancement in Gold-Coated Iron Oxide Nanocrystals. Nano Lett 9:16441650. https://doi.org/10.1021/n1900007k

24. Wang L, Clavero C, Huba Z, et al (2011) Plasmonics and Enhanced Magneto-Optics in Core-Shell Co-Ag Nanoparticles. Nano Lett 11:1237-1240. https://doi.org/10.1021/nl1042243

25. Sohrabi F, Hamidi SM (2017) Fabrication methods of plasmonic and magnetoplasmonic crystals: a review. Eur Phys J Plus 132:15. https://doi.org/10.1140/epjp/i2017-11294-2

26. Yu B, Chen H, Liu Q, et al (2018) Magneto-optical Studies of Noble Metal-Magnetic Dielectric Systems. Plasmonics 13:31-38. https://doi.org/10.1007/s11468-016-0480-2

27. Li D, Tang Z, Chen L, et al (2018) Plasmonics Resonance Enhance Magneto-Optical Effects Through Metallic Sub-wavelength Grating with Bismuth Iron Garnet Slab. Plasmonics 13:55-62. https://doi.org/10.1007/s11468-016-0483-z 
28. Steel MJ, Levy M, Osgood RM (2000) High Transmission Enhanced Faraday Rotation in One-Dimensional Photonic Crystals with Defects. IEEE Photonics Technol Lett 12:1171-1173

29. Yoshimoto T, Goto T, Isogai R, et al (2016) Magnetophotonic crystal with cerium substituted yttrium iron garnet and enhanced Faraday rotation angle. Opt Express 24:8746-8753. https://doi.org/10.1364/OE.24.008746

30. Kharratian S, Urey H, Onbaşlı MC (2019) RGB Magnetophotonic Crystals for High-contrast Magnetooptical Spatial Light Modulators. Sci Rep 9:644. https://doi.org/10.1038/s41598-018-37317-9

31. Da H, Liang G (2011) Enhanced Faraday rotation in magnetophotonic crystal infiltrated with graphene. Appl Phys Lett 98:261915. https://doi.org/10.1063/1.3605593

32. Wan X, Qi MQ, Chen TY, Cui TJ (2016) Field-programmable beam reconfiguring based on digitallycontrolled coding metasurface. Sci Rep 6:20663. https://doi.org/10.1038/srep20663

33. Shaltout AM, Shalaev VM, Brongersma ML (2019) Spatiotemporal light control with active metasurfaces. Science 364:eaat3100. https://doi.org/10.1126/science.aat3100

34. Won $\mathrm{R}$ (2017) The rise of plasmonic metasurfaces. Nat Photonics 11:462-464. https://doi.org/10.1038/nphoton.2017.136

35. Zhang J, ElKabbash M, Wei R, et al (2019) Plasmonic metasurfaces with $42.3 \%$ transmission efficiency in the visible. Light Sci Appl 8:53. https://doi.org/10.1038/s41377-019-0164-8

36. De Marcellis A, Palange E, Janneh M, et al (2017) Design Optimisation of Plasmonic Metasurfaces for MidInfrared High-Sensitivity Chemical Sensing. Plasmonics 12:293-298. https://doi.org/10.1007/s11468-0160263-9

37. Giannini V, Fernández-Domínguez AI, Heck SC, Maier SA (2011) Plasmonic nanoantennas: Fundamentals and their use in controlling the radiative properties of nanoemitters. Chem Rev 111:3888-3912. https://doi.org/10.1021/cr1002672

38. Chin JY, Steinle T, Wehlus T, et al (2013) Nonreciprocal plasmonics enables giant enhancement of thin-film Faraday rotation. Nat Commun 4:1599. https://doi.org/10.1038/ncomms2609

39. Caballero B, García-Martín A, Cuevas JC (2015) Faraday effect in hybrid magneto-plasmonic photonic crystals. Opt Express 23:22238-22249. https://doi.org/10.1364/oe.23.022238

40. Lei C, Chen L, Tang Z, et al (2016) Enhancement of magneto-optical Faraday effects and extraordinary optical transmission in a tri-layer structure with rectangular annular arrays. Opt Lett 41:729-732. https://doi.org/10.1364/ol.41.000729

41. Sadeghi S, Hamidi SM (2018) Enhanced Faraday rotation in one dimensional magneto-plasmonic structure due to Fano resonance. J Magn Magn Mater 451:305-310. https://doi.org/10.1016/j.jmmm.2017.11.080

42. Rahimzadeh Kalaleh Rodriguez S (2012) Coupling Light and Matter in Metallic Nanoparticle Arrays. Eindhoven University of Technology

43. Bhattacharyya S, Ghosh S, Srivastava KV (2017) A Wideband Cross Polarization Conversion Using Metasurface. Radio Sci 52:1395-1404. https://doi.org/10.1002/2017RS006396

44. Zhu W, Yang R, Fan Y, et al (2018) Controlling optical polarization conversion with Ge2Sb2Te5-based phasechange dielectric metamaterials. Nanoscale 10:12054-12061. https://doi.org/10.1039/c8nr02587h

45. Khan MI, Khalid Z, Tahir FA (2019) Linear and circular-polarization conversion in X-band using anisotropic metasurface. Sci Rep 9:4552. https://doi.org/10.1038/s41598-019-40793-2

46. Fan Y, Tu L, Zhang F, et al (2018) Broadband Terahertz Absorption in Graphene-Embedded Photonic Crystals. Plasmonics 13:1153-1158. https://doi.org/10.1007/s11468-017-0615-0

47. Kalish AN, Komarov RS, Kozhaev MA, et al (2018) Magnetoplasmonic quasicrystals: an approach for multiband magneto-optical response. Optica 5:617-623. https://doi.org/10.1364/optica.5.000617

48. Pappas SD, Lang P, Eul T, et al (2019) Broadband enhancement of the magneto-optical activity of hybrid Au 
loaded Bi:YIG. Prepr https//arxiv.org/abs/190511941

49. Gao H, McMahon JM, Lee MH, et al (2009) Rayleigh anomaly-surface plasmon polariton resonances in palladium and gold subwavelength hole arrays. Opt Express 17:2334-2340. https://doi.org/10.1364/oe.17.002334

50. Hayashi H, Iwasa S, Vasa NJ, et al (2002) Characteristics of Bi:YIG magneto-optic thin films fabricated by pulsed laser deposition method for an optical current transformer. Jpn J Appl Phys 41:410-411. https://doi.org/10.1143/JJAP.41.410

51. Bondarev I V., Shalaev VM (2017) Universal features of the optical properties of ultrathin plasmonic films. Opt Mater Express 7:3731-3740. https://doi.org/10.1364/ome.7.003731

52. Kang ESH, Shiran Chaharsoughi M, Rossi S, Jonsson MP (2019) Hybrid plasmonic metasurfaces. J Appl Phys 126:140901. https://doi.org/10.1063/1.5116885

53. Bondarev I V., Mousavi H, Shalaev VM (2018) Optical response of finite-thickness ultrathin plasmonic films. MRS Commun 8:1092-1097. https://doi.org/10.1557/mrc.2018.153

54. Varnavski O, Ramakrishna G, Kim J, et al (2010) Critical Size for the Observation of Quantum Confinement in Optically Excited Gold Clusters. J Am Chem Soc 132:16-17

55. Manjavacas A, García de Abajo FJ (2014) Tunable plasmons in atomically thin gold nanodisks. Nat Commun 5:3548. https://doi.org/10.1038/ncomms4548

56. García de Abajo FJ, Manjavacas A (2015) Plasmonics in atomically thin materials. Faraday Discuss 178:87107. https://doi.org/10.1039/c4fd00216d

57. Shah D, Reddy H, Kinsey N, et al (2017) Optical Properties of Plasmonic Ultrathin TiN Films. Adv Opt Mater 5:1700065. https://doi.org/10.1002/adom.201700065

58. Maniyara RA, Rodrigo D, Yu R, et al (2019) Tunable plasmons in ultrathin metal films. Nat Photonics 13:328333. https://doi.org/10.1038/s41566-019-0366-X

59. Liyanage T, Nagaraju M, Johnson M, et al (2020) Reversible Tuning of the Plasmoelectric Effect in Noble Metal Nanostructures through Manipulation of Organic Ligand Energy Levels. Nano Lett 20:192-200. https://doi.org/10.1021/acs.nanolett.9b03588

60. Johnson PB, Christy RW (1972) Optical Constant of the Nobel Metals. Phys Rev B 6:4370-4379

61. Palik ED (1985) Handbook of Optical Constants of Solids. Academic Press, Orlando

62. Belotelov VI, Doskolovich LL, Kotov VA, et al (2007) Magnetooptical effects in the metal-dielectric gratings. Opt Commun 278:104-109. https://doi.org/10.1016/j.optcom.2007.05.064

63. Belotelov VI, Doskolovich LL, Zvezdin AK (2007) Extraordinary magneto-optical effects and transmission through metal-dielectric plasmonic systems. Phys Rev Lett 98:077401. https://doi.org/10.1103/PhysRevLett.98.077401

64. Dmitriev V, Paixão F, Kawakatsu M (2013) Enhancement of Faraday and Kerr rotations in three-layer heterostructure with extraordinary optical transmission effect. Opt Lett 38:1052. https://doi.org/10.1364/ol.38.001052

65. Zuev VS, Zueva GY (2009) Photon momentum in a surface plasmon. Opt Spectrosc 106:248-251. https://doi.org/10.1134/S0030400X09020167

66. Wiederrecht G (2010) Handbook of Nanoscale Optics and Electronics, First. Amsterdam

67. Safiabadi Tali SA, Zhou W (2019) Multiresonant plasmonics with spatial mode overlap: Overview and outlook. Nanophotonics 8:1199-1225. https://doi.org/10.1515/nanoph-2019-0088

68. Murai S, Verschuuren MA, Lozano G, et al (2013) Hybrid plasmonic-photonic modes in diffractive arrays of nanoparticles coupled to light-emitting optical waveguides. Opt Express 21:4250-4262. https://doi.org/10.1364/oe.21.004250

69. Huang S, Xie Z, Chen W, et al (2018) Metasurface with multi-sized structure for multi-band coherent perfect 
absorption. Opt Express 26:7066-7078. https://doi.org/10.1364/oe.26.007066

70. Drezdzon SM, Yoshie T (2009) On-chip waveguide isolator based on bismuth iron garnet operating via nonreciprocal single-mode cutoff. Opt Express 17:9276-9281. https://doi.org/10.1364/OE.17.009276

71. Kang M-G, Dong Quoc V, Surabhi S, et al (2019) Spectrometer based real-time magnetic Faraday rotation spectroscopy of Bi-YIG thin films. J Magn Magn Mater 482:61-65. https://doi.org/10.1016/j.jmmm.2019.03.035

72. Zeman EJ, Schatz GC (1987) An accurate electromagnetic theory study of surface enhancement factors for Ag, Au, Cu, Li, Na, Al, Ga, In, Zn, and Cd. J Phys Chem 91:634-643. https://doi.org/10.1021/j100287a028

73. Bandurin DA, Svintsov D, Gayduchenko I, et al (2018) Resonant terahertz detection using graphene plasmons. Nat Commun 9:5392. https://doi.org/10.1038/s41467-018-07848-w

74. Mueller JPB, Rubin NA, Devlin RC, et al (2017) Metasurface Polarization Optics: Independent Phase Control of Arbitrary Orthogonal States of Polarization. Phys Rev Lett 118:113901. https://doi.org/10.1103/PhysRevLett.118.113901 